\title{
Advantages of a Centrifugal Pump in the Development of a Swine Model of an Artificial Placenta
}

\author{
Alex J. Charest-Pekeski \\ University of Toronto \\ Steven K.S. Cho \\ University of Toronto \\ Tanroop Aujla \\ University of Toronto \\ Liqun Sun \\ The Hospital for Sick Children \\ Alejandro A. Floh \\ The Hospital for Sick Children \\ Mark J. McVey \\ The Hospital for Sick Children \\ Ayman Sheta \\ The Hospital for Sick Children \\ Marvin Estrada \\ The Hospital for Sick Children \\ Lynn Crawford-Lean \\ The Hospital for Sick Children \\ Celeste Foreman \\ The Hospital for Sick Children \\ Dariusz Mroczek \\ The Hospital for Sick Children \\ Jaques Belik \\ The Hospital for Sick Children \\ Brahmdeep S. Saini \\ University of Toronto \\ Jessie Mei Lim \\ University of Toronto \\ Olivia J. Moir \\ The Hospital for Sick Children \\ Fu-Tsuen Lee \\ University of Toronto \\ Megan Quinn \\ University of South Australia \\ Jack R.T. Darby \\ University of South Australia \\ Mike Seed \\ The Hospital for Sick Children \\ Janna L. Morrison \\ University of South Australia \\ Christoph Haller ( $\square$ christoph.haller@sickkids.ca ) \\ The Hospital for Sick Children
}

\section{Research Article}

Keywords: Artificial placenta, preterm, pig, cannulation, centrifugal pump, oxygenator, tachycardia, hypertension, fetal development 
Posted Date: January 24th, 2022

DOI: https://doi.org/10.21203/rs.3.rs-1251735/v1

License: @) (i) This work is licensed under a Creative Commons Attribution 4.0 International License. Read Full License 


\section{Abstract}

The recent demonstration of normal development of preterm sheep in an artificial extrauterine environment has renewed interest in artificial placenta (AP) systems as a potential treatment strategy for extremely preterm human infants. However, the feasibility of translating this technology to the human preterm infant remains unknown. Here we report the support of 13 preterm fetal pigs delivered at $102 \pm 4$ days (d) gestation, weighing $616 \pm 139 \mathrm{~g}$ with a circuit consisting of an oxygenator and a centrifugal pump, comparing these results with our previously reported pumpless circuit $(n=12 ; 98 \pm 4 d ; 743 \pm 350 \mathrm{~g})$. The umbilical vessels were cannulated, and fetuses were supported for $46.4 \pm 46.8$ hours using the pumped AP versus $11 \pm 13$ hours on the pumpless AP circuit. Upon initiation of AP support on the pumped system, we observed supraphysiologic circuit flows, tachycardia, and hypertension, while animals maintained on a pumpless AP circuit exhibited subphysiologic flows. On the pumped AP circuit, there was a progressive decline in UV flow and oxygen delivery. We conclude that the addition of a centrifugal pump to the AP circuit improves survival of preterm pigs by augmenting UV flow through the reduction of right ventricular afterload. However, we continued to observe the development of heart failure within a matter of days.

\section{Introduction}

In resource rich nations, extreme prematurity, defined as delivery prior to 28 weeks' gestation, remains the leading cause of childhood mortality and morbidity ${ }^{1,2}$. Survival rates decline with decreasing gestational age (GA), with only $6 \%$ surviving at 22 weeks GA compared to $>90 \%$ surviving at 28 weeks $\mathrm{GA}^{3}$. In Canada alone, the economic burden of caring for extremely preterm infants approaches $\$ 600$ million nationally per annum ${ }^{4}$. Unfortunately, despite advances in medical technology and neonatal intensive care, improvements in the outcomes of children born extremely preterm over the last 15 years have been limited $^{5}$.

At the biological limit of viability (22-25 weeks GA), preterm infants commence pulmonary gas exchange during the late-canalicular and early saccular stages of lung development. Exposure to the positive pressure mechanical ventilation and high partial pressure of inspired oxygen required to achieve adequate gas exchange results in cessation of alveolarization and pulmonary microvascular injury, which is associated with high rates of chronic lung disease and pulmonary hypertension ${ }^{6}$. Furthermore, approximately $50 \%$ of children born at the threshold of viability exhibit neurological disabilities at 30 months corrected age, with half of these cases being classified as severe ${ }^{7}$. Despite major advances in the outcomes of prenatal infants resulting from the widespread administration of prenatal steroids and exogenous surfactant, recent improvements in the morbidity and mortality have been more incremental, emphasizing the need for new innovative approaches to supporting the fragile physiology and development of extremely preterm infants ${ }^{1-3}$.

An artificial placenta (AP) represents a novel approach that aims to maintain the innate fetal circulation while promoting normal prenatal development. Gas exchange is achieved with a low-resistance hollow-fiber membrane oxygenator connected to the fetus via the umbilical vasculature, while incubating the fetus in a fluid filled environment. The first attempt to support previable human infants was reported in the late $1950 s^{8}$; with subsequent progress made using animal models. These early experiments were complicated by the development of heart failure and infections and concurrent improvements in conventional neonatal intensive care led to diminished enthusiasm for AP technology ${ }^{8,9,10}$. However, over recent years, several research teams have demonstrated the feasibility of supporting preterm goat and sheep fetuses with a variety of pumpless and pumped arteriovenous and venovenous AP systems using different approaches to establishing vascular access ${ }^{8,9,11-24}$. Two research teams have successfully demonstrated physiologic fetal sheep hemodynamics with normal organ maturation and minimal injury for periods of up to one month on pumpless arteriovenous AP systems ${ }^{17-19,23}$

Despite these advances, AP is yet to be proven in a realistic animal model of the extremely preterm human infant. At a comparable stage of lung development to a previable human fetus, the preterm sheep is approximately twice the weight ${ }^{25,26}$. Fetal size determines important anatomical considerations for establishing an extracorporeal membrane oxygenation (ECMO) system such as blood vessel diameter and blood pressure. In addition, fetal sheep possess two umbilical arteries (UA) and two umbilical veins (UV). This may allow a more stable transition to the AP circuit using one pair of UA and UV while maintaining native gas exchange via the placenta using the other pair of umbilical vessels, thus minimizing the cessation of oxygenation from the mother ${ }^{27,28}$. By contrast, human and pig fetuses usually have one UV and two UAs, which may represent a more technically challenging approach to the initiation of AP support.

We have previously proposed ${ }^{27}$ that Yucatan miniature pigs delivered at approximately 95 days gestation represent a realistic model of human infants born at the lower limit of viability in terms of their body weight and stage of lung development ${ }^{25}$. We recently demonstrated the feasibility of cannulating the umbilical vessels of preterm minipigs and transferring them to an AP system consisting of a warm, fluid environment and a pumpless ECMO circuit comprising a commercial neonatal oxygenator ${ }^{27}$. However, our experiments were characterized by subphysiologic circuit flows, tachycardia, and the development of hydrops. Echocardiography further revealed evidence of diminished right ventricular function, which we attributed to excessive ventricular afterload resulting from the large priming volume of the circuit and small umbilical cannulas ${ }^{27}$. We hypothesized that the addition of a pump might improve the hemodynamics of our animals on the circuit by reducing afterload through the 
generation of negative pressure downstream of the umbilical arterial cannulas, thereby improving venous return and cardiac output. Herein, we sought to investigate the hemodynamics of a pumped AP system, comparing our findings to those obtained using the pumpless circuit ${ }^{27}$.

\section{Methods}

\section{Experimental groups.}

The results presented in this report are comprised from fetal pigs studied in utero and while maintained using a pumped AP circuit and of our previously published findings using a pumpless AP system ${ }^{27}$. Fetal Large White Landrace pigs were studied in utero at 98,106 , and 112 days GA ( $n=24$ ) and Yucatan miniature pigs at $107 \pm 3$ days $(n=16)$, providing reference physiologic data regarding heart rate (HR), blood pressure, blood gases, electrolytes, lactate and glucose concentrations, and UV flow ${ }^{27}$. Fetal Yucatan miniature pigs $(n=13)$ were maintained using an umbilical arteriovenous AP circuit consisting of a centrifugal pump and oxygenator and using a pumpless AP circuit $(n=12)^{27}$. We included AP subjects that survived on the system for a minimum of 3 hours.

\section{Animals and approvals.}

In utero BP, HR, blood gases, electrolytes, glucose, and lactate measurements were obtained in White Landrace Cross sows ( $\mathrm{n}=6$; term = 115 days) at the Preclinical Imaging and Research Laboratories, South Australian Health and Medical Research Institute (SAHMRI). All procedures were approved by the SAHMRI Animal Ethics Committee 27 . Sows were individually housed with environmental and social enrichment. The AP experiments were conducted in the Lab Animal Services (LAS) facility at The Hospital for Sick Children (SickKids), Peter Gilgan Center for Research and Learning in Toronto, Ontario. All maternal and fetal surgeries were approved by the SickKids Animal Care Committee and all procedures complied with the Canadian Council on Animal Care, Ontario Ministry of Agriculture, Food and Rural Affairs, Animals for Research Act guidelines, and the Care and Use of Animals for Scientific Purposes. All research and LAS staff members acted in accordance with ARRIVE guidelines proposed by Kilkenny et al. ${ }^{54}$. Pregnant Yucatan miniature pigs ( $n=46$; term = 115 days) were acquired from Memorial University of Newfoundland and Sinclair Bioresources and transported as per the Health of Animals Act of Canada ${ }^{27}$. Yucatan pigs were housed in pairs for at least two weeks prior to surgery to increase socialization between the animals and allow for acclimation to human handling and to their new environment. Sows were provided with ad libitum food and water, and environmental enrichments as per SickKids standard operating procedures.

\section{Protocol for in utero studies.}

Large White Landrace Cross gilts ( $\mathrm{n}=6$; $98 \pm 7$ days GA; term = 115 days) were anaesthetised with an intramuscular injection (I.M.) of 20 mg/kg ketamine and inhalation of isoflurane. Gilts were intubated and general anaesthesia was maintained using isoflurane with $2 \mathrm{~L} / \mathrm{min} \mathrm{O}_{2}$ and $4 \mathrm{~L} / \mathrm{min}$ medical air. Gilts were positioned on the operating table on their backs, an incision was made along the abdomen, the uterus was incised, and a fetal head was exposed. Fetal pigs $(n=24)$ were cannulated via the UV, and venous blood was sampled for partial pressure of oxygen $\left(\mathrm{PO}_{2}\right)$, partial pressure of carbon dioxide $\left(\mathrm{PCO}_{2}\right)$, oxygen saturation $\left(\mathrm{SO}_{2}\right), \mathrm{pH}$, hemoglobin $(\mathrm{Hb})$, bicarbonate $\left(\mathrm{HCO}_{3}{ }^{-}\right)$, base excess $(\mathrm{BE})$, sodium $\left(\mathrm{Na}^{+}\right)$, potassium $\left(\mathrm{K}^{+}\right)$, and calcium $\left(\mathrm{Ca}^{2+}\right)$ as previously described ${ }^{27,28}$. In a subset of fetuses $(\mathrm{n}=21,105 \pm 7$ days $\mathrm{GA})$, the carotid artery $(\mathrm{CA})$ was instrumented and fetal BP, and HR were measured and continuously recorded in LabChart 8 Pro (ADInstruments Inc., Colorado Springs, USA) ${ }^{27}$. Following in utero experiments gilts and their fetal pigs were humanely euthanized with an intravenous overdose of sodium pentobarbital (Virbac, New South Wales, Australia).

\section{Surgical protocol for pumped AP studies.}

Pregnant Yucatan pigs ( $\mathrm{n}=19 ; 101 \pm 4$ days GA; term $=115$ days) were anaesthetized with an I.M. injection of $10 \mathrm{mg} / \mathrm{kg}$ ketamine hydrochloride, $0.20 \mathrm{mg} / \mathrm{kg}$ acepromazine, and $0.015 \mathrm{mg} / \mathrm{kg}$ atropine sulfate (CDMV Inc., Saint-Hyacinthe, Canada), with maintenance of general anaesthesia with inhalation of 2-3\% isoflurane (Fresenius Kabi Canada, Toronto, Canada). To prevent aortocaval compression, anaesthetized sows were positioned on the operating table in the left lateral position. Umbilical blood flow was measured in utero in 4 Yucatan sows in Toronto using cine phase contrast MRI as described in our previous publication. ${ }^{27}$ A lower antero-lateral laparotomy was performed for a caesarean section. Following the delivery of all fetal pigs, sows were humanely euthanized with 106 mg/kg Euthanyl (CDMV Inc., Saint-Hyacinthe, Canada).

\section{Fetal surgical procedures.}

In a subset of fetuses ( $\mathrm{n}=11 / 13$ successful pumped AP experiments), a small incision was made along the right side of the neck to expose the jugular vein (JV) and $\mathrm{CA}^{28,55}$. A size-matched custom-made PVC tubing was then inserted in the JV and CA for monitoring of central venous pressure (CVP) and fetal mean arterial pressure (MAP), respectively. The tubing was stabilized using silk sutures, and the neck incision sutured closed. Fetuses were delivered to minimize excessive torsion and stretching of the umbilical cord and were positioned on the maternal abdomen and subsequently weighed. Fetal pigs were then anaesthetized using an I.M. injection of $1 \mathrm{mg} / \mathrm{kg}$ rocuronium bromide (Sandoz Inc., Mississauga, Canada), and $5 \mathrm{mg} / \mathrm{kg}$ ketamine hydrochloride (CDMV Inc., Saint-Hyacinthe, Canada). Fetal normothermia was maintained by continuously 
bathing the fetus and the umbilical cord in warmed normal saline. The umbilical cord was treated with a topical application of $100 \mathrm{mg} / \mathrm{kg}$ papaverine hydrochloride (Sandoz Inc., Boucherville, Canada).

In all AP studies, umbilical catheters were then placed in both UAs and the UV (12-gauge and 10-gauge custom-made cannulas, respectively), and secured into place using silk sutures (Ethicon Inc., New Jersey, USA) at the insertion of the cannula within the vessel. In smaller fetuses, UA cannulas were downsized to $14 \mathrm{GA}$ angiocaths (Becton Dickinson Canada Inc., Mississauga, Canada). Cannulas were then connected to the AP circuit described below, via modified $1 / 4$ " perfusion adapters (Medtronic of Canada Ltd., Brampton, Canada) to facilitate the initiation of UV flows. Re-positioning of the umbilical catheters was performed as needed to assist in the establishment of flow.

\section{AP circuit design.}

The arteriovenous AP circuit consisted of a commercially available centrifugal pump, connected to a low-resistance membrane oxygenator, and modified commercially available intravenous umbilical arterial and venous cannulas via $1 / 4$ " internal diameter $x 1 / 16^{\prime \prime}$ wall thickness, $3 / 16^{\prime \prime} \times 1 / 16$ " TYGON PVC tubing with P.h.i.s.i.o coating (LivaNova PLC., London, England). In two experiments, a commercially available cardiopulmonary bypass oxygenator was used (Table 2); however, this was subsequently changed to another commercially available ECMO oxygenator ( $\mathrm{n}=11$; Table 2). Total priming volumes for these neonatal membrane oxygenators were 40 and $65 \mathrm{ml}$, respectively. Circuits were then initially primed with plasmalyte (Baxter Inc., Mississauga, Canada) and then replaced with heparinized maternal blood, which was continuously recirculated to prevent clotting within the circuit and warm the blood prior to connection to the fetus. UA blood passed through the oxygenator inlet, exiting through the outlet before returning to the heart via the UV (Figure 1). The sweep gas supplied the oxygenator with a mixture of medical air and oxygen and was titrated to achieve normal physiologic levels of $\mathrm{UV} \mathrm{PO}_{2}$ of $35-40 \mathrm{mmHg}$ and a $\mathrm{PCO}_{2}$ of $50-60 \mathrm{mmHg}^{29}$ based on in utero fetal pig data.

\section{Fluid incubation}

Following cannulation, fetal pigs were enclosed in a custom-made silicone infused thermoplastic polyurethane film 'biobag'. Approximately 2-4 litres of lactated ringer (LR; Baxter Inc., Mississauga, Canada) crystalloid solution was warmed to $39 \pm 1^{\circ} \mathrm{C}$ in a large fluid reservoir and circulated via the inflow and outflow ports of the Biobag every 10 hours. The Biobag was equipped with a temperature port that facilitated monitoring of fluid temperature using a temperature probe (ADInstruments Inc., Colorado Springs, USA). The biobag was covered to prevent transmission of light to the fetus to better simulate uterine conditions and maintained at an appropriate temperature using a contact heat pad underneath the biobag and an overhead heater.

\section{Fetal pig maintenance on the AP circuit.}

Following cannulation and transition to the AP, fetal pigs received a maintenance infusion of $6 \mu \mathrm{g} / \mathrm{kg} / \mathrm{h}$ prostaglandin E1 (Pfizer Canada Inc., Kirkland, Canada) with 100 units/kg/h heparin (Fresenius Kabi Canada, Toronto, Canada) to maintain patency of the ductus arteriosus, and prevent clotting in the AP circuit ${ }^{19}$. Dextrose $(418 \mathrm{mg} / \mathrm{kg} / \mathrm{h}$; Pfizer Canada Inc., Kirkland, Canada) was delivered via the circuit for the first 8 hours of AP support, which was then exchanged for total parenteral nutrition solution with the goal of supporting fetal energy requirements and maintaining fetal glucose concentrations of $\geq 100 \mathrm{mg} / \mathrm{dL}$. Calcium chloride ( $80 \mathrm{mg} / \mathrm{kg}$; Omega Laboratories Ltd., Montreal, Canada) and heparin (100 units $/ \mathrm{kg}$ ) were dosed empirically to achieve a target $\mathrm{Ca}^{++}$concentration of $\geq 1.4 \mathrm{mmol} / \mathrm{L}$, and an activated clotting time (ACT) of $250-300$ seconds, respectively. In early experiments ( $n=7 / 13$ successful experiments), $6 \mathrm{mg} / \mathrm{kg} / \mathrm{h}$ of papaverine was administered I.V. to prevent vasospasm of the umbilical vessels and augment circuit flows. In a subset of experiments $(n=3 / 13)$, this was subsequently changed to a maintenance infusion of $30 \mu \mathrm{g} / \mathrm{kg} / \mathrm{h}$ milrinone lactate (Aurobindo Pharma, Hyderabad, India). Reconstituted hydrocortisone (8 mg/kg/day, Pfizer Canada Inc., Kirkland, Canada) and a broad-spectrum empirical antibiotic (piperacillin/tazobactam; 300 mg/kg/day, Sandoz Inc., Boucherville, Canada) was administered I.V., every 6 hours, and 8 hours, respectively. Whole maternal blood was transfused (10 ml/kg) to replete circulating blood volume following circuit phlebotomy. In addition, rocuronium bromide $(1 \mathrm{mg} / \mathrm{kg}$ ) and ketamine hydrochloride (5 mg/kg) were given for excessive fetal movements or perceived fetal agitation. Albumin (25\%; CSL Behring Canada Inc., Ottawa, Canada) and furosemide (0.5 mg/kg; Pfizer Canada Inc., Kirkland, Canada) were given intermittently during longer experiments to address perivascular edema and hydrops fetalis.

Fetal $\mathrm{CA}$, and umbilical arterial and venous blood gases including $\mathrm{PO}_{2}, \mathrm{PCO}_{2}, \mathrm{pH}, \mathrm{Hb}, \mathrm{HCO}_{3}{ }^{-}, \mathrm{SO}_{2}, \mathrm{BE}, \mathrm{Na}^{+}, \mathrm{K}^{+}$, lactate, glucose and $\mathrm{ACT}$ were sampled every 1-3 hours and analyzed using a handheld blood analyzer (Abbott Point of Care Inc., Nepean, Canada). Fetal oxygen delivery (DO ${ }_{2}$ ) and consumption $\left(\mathrm{VO}_{2}\right)$ were calculated based on the combination of UV and UA oxygen carrying capacity and indexed UV flow indexed to fetal weight (measured at surgery).

\footnotetext{
$\mathrm{DO}_{2}=$ Indexed UV flow $*\left(1.36 *[\mathrm{Hb}] * \mathrm{UV} \mathrm{\textrm {SO } _ { 2 }}\right)$

$\mathrm{VO}_{2}=$ Indexed UV flow $*(\mathrm{UV} \mathrm{SO} 2-\mathrm{UA} \mathrm{SO})^{*} 1.36 *[\mathrm{Hb}]$
}

Oxygen extraction fraction $(\%)=\left(\left(\left(\mathrm{UV} \mathrm{SO} \mathrm{S}_{2}-\mathrm{UA} \mathrm{SO}_{2}\right)\right) /\left(\mathrm{UV} \mathrm{SO}_{2}\right)\right) * 100$ 
UV flow was continuously measured using a HXL 3/16" tubing flow probe (Transonic) and CVP, fetal BP, pre-pump circuit pressure, pre-oxygenator circuit pressure, and post-oxygenator circuit pressure were measured using Deltran fluid filled blood pressure transducers (ADInstruments Inc., Colorado Springs, USA). Data were sampled at $1000 \mathrm{~Hz}$, digitized, and continuously recorded using LabChart Pro 8 (ADInstruments Inc., Colorado Springs, USA). At the end of each study, the data was extracted in consecutive 30-second intervals and analyzed in Excel (Microsoft Corporation, Washington, USA).

\section{Quantification of UV blood flow in utero using MRI: 3-D Volumetry and cine phase-contrast.}

In utero fetal weight and UV flow were measured in sixteen fetuses from 4 pregnant Yucatan pigs ( $=4$; gestational age $107 \pm 3$ days $\mathrm{GA}$ ) as previously described using a 3 Tesla magnetic resonance imaging (MRI) system ${ }^{27}$.

\section{Statistical analysis.}

Comparisons of anthropometric data between pumpless and pumped AP circuits were analyzed using a two-way ANOVA with Bonferroni correction for multiple comparisons. Comparison of the duration of AP support between pumpless and pumped circuits was analyzed using a Mann-Whitney $U$ test. Changes in temperature, HR, and UV flow between pumpless and pumped AP circuits over the first 3 hours of support were analyzed using a mixed-effect model, with a Bonferroni correction for multiple comparisons. Differences in mean indexed and absolute UV flow and HR between in utero, pumpless and pumped AP circuits were compared using a repeated measures one-way ANOVA with a Bonferroni correction for multiple comparisons. Analysis of fetal HR and indexed UV flow were performed using a linear regression. Comparisons of mean blood gases, electrolytes, lactate, and glucose concentrations between animals studied in utero, or on pumpless and pumped AP circuits were analyzed using a repeated measure one-way ANOVA, and a Kruskal-Wallis test (when appropriate) with Bonferroni correction for multiple comparisons. UV flow, HR, fetal BP, CVP, and temperature are presented in 5-minute averages. Time post-cannulation versus UV flow is presented in 1 hour averages and analyzed using a one-way ANOVA with Bonferroni correction for multiple comparisons. Differences in MAP between fetal pigs studied in utero and those maintained using a pumped AP were analyzed using a mixed-effect model with Bonferroni correction for multiple comparisons. Indexed UV flow versus oxygen extraction was analysed using a linear regression. * $P<0.05$ was considered statistically significant. All statistical analyses were performed using Prism 9 (GraphPad, San Diego, USA). Data are presented as mean \pm standard deviation (SD), unless otherwise indicated.

\section{Results}

\section{The addition of a centrifugal pump improves successful transition to AP and support times.}

Nineteen sows and 89 fetal pigs were used for pumped AP experiments (Table 1). A total of 32 fetal pigs were cannulated and transitioned to the AP. Types of perioperative complications preventing us from maintaining AP support were similar to those experienced in the pumpless AP circuit group and included the inability to establish circuit flows upon connection to the circuit $(n=11)$, accidental decannulation $(n=4)$, persistent vasospasm of the umbilical cord $(n=3)$, and excessive spiralling of the umbilical cord, which prevented cannulation or adequate cannula position ( $n=1$; Table 1). We successfully maintained 13 fetal pigs at an initial GA of $102 \pm 4$ days (range $=93-107$ days) with a body weight of $616 \pm 139 \mathrm{~g}$ (range $=390-820 \mathrm{~g}$ ) on a pumped AP circuit for $46.4 \pm 46.8$ hours (range $=3.4-177.8$ hours). This represented a significant improvement in the duration of support over the 12 fetal pigs ( $98 \pm 4$ days GA; $743 \pm 350 \mathrm{~g} ; 11 \pm 13$ hours) maintained using a pumpless AP circuit ( $P=0.009$; Table 1 and Figure 2). There was also a marked improvement in the rate of successful AP runs per litter using a pumped AP circuit compared to the pumpless AP circuit (62\% pumped AP vs. $28 \%$ pumpless AP; Table 1$)$. Successfully supported fetuses were similar in weight and GA in both pumped and pumpless groups (616 $\pm 139 \mathrm{~g}$ vs. $743 \pm 350 \mathrm{~g}, P=0.41 ; 102 \pm 4$ days vs. $98 \pm 4$ days, $P=0.24)$. On the pumped and pumpless AP circuits, fetal body weights were similar between successfully and unsuccessfully supported animals $(616 \pm 139 \mathrm{~g}$ vs. $523 \pm 145 \mathrm{~g}, P=0.47 ; 743 \pm$ $350 \mathrm{~g}$ vs. $644 \pm 184 \mathrm{~g}, P=0.53)$. However, unsuccessfully supported fetuses or fetuses that we did not attempt to cannulate were significantly smaller in the pumped group compared to the pumpless group ( $523 \pm 145 \mathrm{~g}$ vs. $644 \pm 184 \mathrm{~g}, P=0.04 ; 557 \pm 124 \mathrm{~g}$ vs. $892 \pm 362 \mathrm{~g}, P<0.0001$ ). Fetal weight at the time of termination of the AP study was significantly higher compared to pre-cannulation weight. Fetal weight gain correlated strongly with length of AP support $(r=0.89)$, with weight at the end of the experiment exceeding the expected growth trajectory of these fetuses. Individual data on supported fetuses, support times, and reasons for termination are shown in Table 2.

\section{Table 1. Summary of fetal pigs from pumped and pumpless AP circuits ${ }^{27}$.}




\begin{tabular}{|c|c|c|c|c|c|c|c|c|c|c|c|}
\hline & \multicolumn{4}{|c|}{ Pumped AP circuit } & \multicolumn{7}{|c|}{ Pumpless AP circuit } \\
\hline & \multicolumn{4}{|c|}{ Intervention } & \multicolumn{7}{|c|}{ Intervention } \\
\hline Parameter & \multicolumn{2}{|c|}{$\begin{array}{l}\text { Maintained } \\
\text { on AP }\end{array}$} & $\begin{array}{l}\text { Cannulated } \\
\text { but not } \\
\text { maintained } \\
\text { on AP }\end{array}$ & $\begin{array}{l}\text { Did not } \\
\text { attempt } \\
\text { to } \\
\text { cannulate }\end{array}$ & \multicolumn{2}{|c|}{$\begin{array}{l}\text { Maintained } \\
\text { on AP }\end{array}$} & $\begin{array}{l}\text { Cannulated } \\
\text { but not } \\
\text { maintained } \\
\text { on AP }\end{array}$ & \multicolumn{4}{|l|}{$\begin{array}{l}\text { Did not } \\
\text { attempt } \\
\text { to } \\
\text { cannulate }\end{array}$} \\
\hline \multirow[t]{2}{*}{$\mathrm{N}$} & \multirow[t]{2}{*}{13} & \multirow{2}{*}{$\begin{array}{l}\text { AP } \\
\text { success } \\
\text { rate per } \\
\text { litter } \\
(\%)\end{array}$} & \multirow[t]{2}{*}{19} & \multirow[t]{2}{*}{57} & \multirow[t]{2}{*}{12} & $\begin{array}{l}\text { AP } \\
\text { success } \\
\text { rate per } \\
\text { litter } \\
(\%)\end{array}$ & \multirow[t]{2}{*}{56} & \multirow[t]{2}{*}{9} & \multicolumn{3}{|c|}{$\begin{array}{l}\text { P-values: Pumpless vs. Pumped AP } \\
\text { circuit (ANOVA results) }\end{array}$} \\
\hline & & & & & & 28 & & & $\begin{array}{l}\text { Circuit } \\
\text { type }\end{array}$ & Intervention & Interaction \\
\hline $\begin{array}{l}\text { Body } \\
\text { weight (g) }\end{array}$ & \multicolumn{2}{|c|}{$\begin{array}{l}616 \pm 139 \\
(390-890)\end{array}$} & $\begin{array}{l}523 \pm 145 \\
(244-710) \text { * }\end{array}$ & $\begin{array}{l}557 \pm \\
124(372- \\
941 ; n= \\
24)^{\star}\end{array}$ & \multicolumn{2}{|c|}{$\begin{array}{l}743 \pm 350 \\
(500-1500 ; n \\
=7) a b\end{array}$} & $\begin{array}{l}644 \pm 184 \\
(343-1390 \\
\mathrm{n}=52) a\end{array}$ & $\begin{array}{l}892 \pm \\
362(531- \\
1470) \boldsymbol{b}\end{array}$ & $<0.0001$ & 0.002 & 0.02 \\
\hline GA (days) & \multicolumn{2}{|c|}{$\begin{array}{l}102 \pm 4 \text { (93- } \\
107)\end{array}$} & $\begin{array}{l}103 \pm 4 \\
(93-108)\end{array}$ & $\begin{array}{l}102 \pm 5 \\
(93-108)\end{array}$ & \multicolumn{2}{|c|}{$\begin{array}{l}98 \pm 4 \text { (93- } \\
107)\end{array}$} & $\begin{array}{l}101 \pm 7 \\
(93-112)\end{array}$ & $\begin{array}{l}101 \pm 7 \\
(97-110)\end{array}$ & 0.04 & 0.34 & 0.60 \\
\hline
\end{tabular}

Two-way ANOVA. Multiple comparisons for fetal body weight and GA within pumped and pumpless AP circuits and between the intervention groups: $\boldsymbol{a} \boldsymbol{b} P<0.05 .{ }^{*} P<0.05$ body weight is significantly different between pumped and pumpless AP circuits within intervention groups (i.e., body weight maintained on a pumped AP circuit vs. body weight maintained on a pumpless AP circuit). Interpretation of numbers for multiple comparisons: a represents a statistically significant difference compared with $b$.

Table 2. Summary data of 13 fetal pigs successfully cannulated and maintained using a pumped AP circuit. Data are presented by increasing GA and are expressed as mean \pm SD.

\begin{tabular}{|llllllll|}
\hline $\begin{array}{l}\text { GA } \\
\text { before } \\
\text { (days) }\end{array}$ & $\begin{array}{l}\text { Weight } \\
\text { before } \\
\mathbf{( g )}\end{array}$ & $\begin{array}{l}\text { Duration on } \\
\text { AP } \\
\text { (hours) }\end{array}$ & $\begin{array}{l}\text { GA } \\
\text { after } \\
\text { (days) }\end{array}$ & $\begin{array}{l}\text { Weight after } \\
(\mathbf{g})\end{array}$ & $\begin{array}{l}\text { Sex } \\
(\mathbf{M},\end{array}$ & Reason for termination & Oxygenator \\
\hline 93 & 390 & 36.7 & 95 & 450 & F & Fetal movement occluded UV flow & 0 \\
\hline 98 & 450 & 3.4 & 98 & 450 & M & Heart failure & 0 \\
\hline 100 & 505 & 14.1 & 101 & NA & M & Mechanical failure (pump) & 0 \\
\hline 100 & 520 & 15.1 & 101 & 620 & M & Heart failure & BP \\
\hline 100 & 590 & 49.4 & 102 & 750 & F & Heart failure & 0 \\
\hline 101 & 762 & 70.0 & 104 & 1190 & M & Mechanical failure (oxygenator) & BP \\
\hline 102 & 480 & 177.8 & 109 & 1176 & M & Hydrops, cardiac dysfunction & 0 \\
\hline 105 & 610 & 68.5 & 108 & NA & NA & Mechanical failure (oxygenator) & 0 \\
\hline 105 & 676 & 17.1 & 105 & NA & M & Fetal movement occluded UV flow & 0 \\
\hline 105 & 690 & 53.5 & 108 & NA & M & Fetal movement occluded UV flow & 0 \\
\hline 105 & 730 & 4.2 & 105 & 730 & M & Thrombosis development in circuit & 0 \\
\hline 105 & 780 & 21.1 & 106 & 879 & M & $\begin{array}{l}\text { Equipment failure (sweep-gas } \\
\text { supply) }\end{array}$ \\
\hline 107 & 820 & 72.5 & 110 & 876 & F & Fetal movement occluded UV flow & 0 \\
\hline $102 \pm 4$ & $616 \pm 139$ & $46.4 \pm 46.8$ & $104 \pm 4$ & $792 \pm 272(n=$ & & & \\
\hline
\end{tabular}

Abbreviations: NA, not available; $\mathrm{M}$, male; F, female; GA, gestational age; $\mathrm{O}$, commercially available ECMO oxygenator; $\mathrm{BP}$, commercially available bypass oxygenator. 
Animals on a pumped AP circuit maintained a physiologic core body temperature throughout the experiment. The temperature was lower in fetal pigs supported on a pumped system than pigs supported on a pumpless system $(P<0.0001$, Figure $3 \mathrm{~A})$. UV flow rates of fetuses supported by the pumped AP circuit were comparable to in utero controls, while UV flow on the pumpless AP system was subphysiologic (in utero; $108 \pm 24 \mathrm{ml} / \mathrm{min}$ vs. pumped; $87 \pm 28 \mathrm{ml} / \mathrm{min}$ vs. pumpless; $70 \pm 18 \mathrm{ml} / \mathrm{min}, P=0.001 ;$ in utero; $173 \pm 45 \mathrm{ml} / \mathrm{min} / \mathrm{kg}$ vs. pumped; $143 \pm 40 \mathrm{ml} / \mathrm{min} / \mathrm{kg} \mathrm{vs}$. pumpless; $97 \pm 39 \mathrm{ml} / \mathrm{min} / \mathrm{kg}, P=0.005$; Figure $3 \mathrm{C}, \mathrm{E}$ and F). Absolute UV flow measured within the first 3 hours post-cannulation was positively correlated to HR in pumped AP circuits $\left(r^{2}=0.44 ; P<0.0001\right.$; Figure 3G), but negatively correlated in fetuses maintained on a pumpless circuit $\left(r^{2}=0.45 ; P<\right.$ 0.0001; Figure 3G), with differences between the slopes being extremely significant $(P<0.0001)$. Figures 5A-M demonstrate the changes in UV flow, HR, temperature, MAP, and CVP over time for the 13 fetal pigs successfully maintained using a pumped AP circuit. Upon initiation of AP support, we observed supraphysiologic UV flow, despite a low pump rate setting (Figure 5A-N). In longer trial runs, this period of supraphysiologic UV flow persisted for approximately 6 hours, then decreasing to subphysiologic flow, plateauing at $\sim 12$ hours of support (Figure 50 ). In several experiments $(n=8 / 13$ ) excessive spasmodic fetal movements resulted in a complete cessation of UV flow and consequently led to the termination of the experiment (Table 2). In four studies, development of a thrombosis in one of the UAs in the first 12 hours of support was associated with a significant reduction in UV flow.

Fetal pigs were tachycardic for a large proportion of the AP studies (Figure 3B and D; and Figures $5 \mathrm{~A}-\mathrm{M}$ ). Both pumped (205 \pm 28 bpm; $P<0.0001$; Figure 3D) and pumpless (206 \pm 38 bpm; $P<0.0001$; Figure 3D) fetal pig groups were similarly tachycardic throughout the experiment compared to in utero controls $(130 \pm 10 \mathrm{bpm}$; Figure $3 \mathrm{D})$. Compared to in utero control animals, fetal pigs were hypertensive on the pumped AP system $(P=$ 0.013; Figure 6). Approximately 20 minutes after initiation of a milrinone infusion, there was a marked increase in UV flow, followed by a steady decline over a 3 hour period (Figure 7A). MAP and HR decreased following the start of the milrinone infusion, which persisted for $\sim 1$ hour before returning to a steady state (Figure 7B and $\mathrm{C}$.).

Upon initiation of AP support, fetal oxygen delivery was physiologic ${ }^{29,30}$. We observed a decline in oxygen delivery and increase in oxygen consumption over time (Figure $8 \mathrm{~A})$. UV flow and oxygen extraction fraction correlated negatively $\left(\mathrm{r}^{2}=0.45 ; P=0.0003 ;\right.$ Figure $\left.8 \mathrm{~B}\right)$.

\section{Fetal blood gas analysis indicates supraphysiologic oxygen tension and fetal anemia}

While there was no significant difference in $\mathrm{SO}_{2}$ between fetal pigs studied in utero and those maintained using a pumped or pumpless AP circuit (Figure 4A), both AP circuits resulted in a significantly higher $\mathrm{PO}_{2}$ compared to in utero controls (in utero; $53 \pm 16 \mathrm{mmHg}$ vs. pumped; $141 \pm 129$ $\mathrm{mmHg}, P=0.0001$, vs. pumpless; $280 \pm 176 \mathrm{mmHg}, P=0.003$, Figure 4B). Conversely, $\mathrm{PCO}_{2}$ was significantly lower in the pumped AP group compared to in utero controls (in utero; $67 \pm 11 \mathrm{mmHg}$ vs. pumped; $49 \pm 11 \mathrm{mmHg}, P=0.0009$, Figure $4 \mathrm{E}$ ). Animals on the pumped AP were significantly more alkalotic than both pumpless AP fetuses and in utero controls (in utero; $7.29 \pm 0.08$ vs. pumped; $7.36 \pm 0.07, P=0.03$, vs. pumpless; $7.30 \pm 0.06, P=0.03$, Figure 4F). BE and lactate were similar between groups (Figure 4G, H). We identified significantly lower hematocrit (in utero; $28 \pm 3 \%$; vs. pumped; $24 \pm 3 \%, P=0.02$, Figure 4C) and hemoglobin in the pumped AP group (in utero; $96 \pm 9 \mathrm{~g} / \mathrm{L}$ vs. pumped: $82 \pm 11$ $\mathrm{g} / \mathrm{L}, P=0.01$, Figure 4D), as well as higher $\mathrm{Na}^{+}$in both AP supported groups compared to intrauterine fetuses (in utero; $126 \pm 2 \mathrm{mmol} / \mathrm{L}$ vs. pumped; $133 \pm 5 \mathrm{mmol} / \mathrm{L}, P<0.0001$; vs. pumpless; 133 vs. $3 \mathrm{mmol} / \mathrm{L}, P<0.0001$, Figure $4 \mathrm{~L})$. Ca ${ }^{2+}$ concentrations were subphysiologic in both pumped and pumpless groups (in utero; $1.56 \pm 0.087$ vs. pumped; $1.44 \pm 0.092 \mathrm{mmol} / \mathrm{L}, P=0.003, \mathrm{vs}$. pumpless; $1.29 \pm 0.21 \mathrm{mmol} / \mathrm{L}, P=0.001$, Figure $4 \mathrm{~K})$. Glucose, administered continuously either directly or as part of total parenteral nutrition, was significantly higher in both AP groups, compared to in utero controls (in utero; $2.1 \pm 0.43 \mathrm{mmol} / \mathrm{L}$ vs. pumped; $8.5 \pm 4.8 \mathrm{mmol} / \mathrm{L}, P<0.0001$, vs. pumpless; $9.8 \pm 5.8 \mathrm{mmol} / \mathrm{L}, P<0.0001$, Figure $4 \mathrm{I}$ ). There was no difference in $\mathrm{K}^{+}$concentrations between in utero fetal pigs and those maintained using a pumped and pumpless AP circuit (Figure 4J).

\section{Discussion}

To our knowledge, this is the first study to examine the hemodynamics and blood gas status of preterm pigs supported using an AP system incorporating a small centrifugal pump. We previously showed the feasibility of supporting our animal model using a pumpless AP circuit connected to the fetal circulation via the umbilical arteries and vein ${ }^{27}$; however, our experiments were characterized by significant hemodynamic decompensation within hours. We attributed this to afterload imbalance, elicited by supraphysiologic circuit resistance and further exacerbated by impaired umbilical venous return, which we surmised resulted in higher sympathetic tone, further exacerbating the increase in afterload ${ }^{27}$. Although other research groups have highlighted potential limitations of pumped ECMO circuits, including an increased risk of pump-induced hemolysis ${ }^{17}$, afterload imbalance, myocardial strain, and impaired autoregulation of UV blood flow ${ }^{31}$, we hypothesized that the addition of a small pump might reduce right ventricular afterload and sustain adequate UV flow, thereby improving survival on the AP. The present study examined differences in hemodynamics, blood gases, electrolytes, biochemistry, and survival of fetal pigs supported on a pumped versus pumpless AP system $^{27}$. The increase in AP support to $46.4 \pm 46.8$ hours represented a significant increase in survival over fetal pigs supported without a pump possibly due to more reliable and physiologic UV flow compared to a pumpless system. Despite the pump support, following a period of supraphysiologic UV flow, the animals continued to experience a slower but consistent decline in UV flow and the development of signs of 
circulatory deterioration. We have yet to establish an AP system capable of meeting the long-term physiologic needs of animals that are similar in size to the extremely preterm human infants that are the proposed target of this approach.

In a recent report, Partridge et al. ${ }^{17}$ demonstrated AP support of fetal sheep weighing $1-2 \mathrm{~kg}$ using a pumpless system for up to 28 days without the use of vasopressors. Using a similar AP circuit, Usada et al. ${ }^{18}$ reported survival of fetal sheep for up to one week. To investigate the potential utility of AP systems for extremely preterm infants born at 22-25 weeks GA, two research groups cannulated sheep born at 85-95 days GA weighing $\sim 0.5-0.8 \mathrm{~kg}$. Although cannulation was technically feasible, one study reported the development of hydrops fetalis following 5-8 days of support ${ }^{32}$, whereas the other required the use of aggressive pharmacological interventions in order to achieve hemodynamic stability ${ }^{19}$. Although fetal sheep delivered at 85-95 days GA share similar body weight to human fetuses delivered at the biological limit of viability, they are developmentally immature and their pulmonary development is analogous to a human fetus delivered at 18 weeks $\mathrm{GA}^{10,26,33,34}$. By contrast, fetal minipigs delivered at 93-107 days GA weighing 0.39-0.82 kg are in their canalicular and saccular stages of lung development and comparable to preterm human fetuses delivered at 22-28 weeks GA. Thus, fetal pigs delivered at 95 days GA would therefore be equivalent in body weight and lung maturity to human fetuses born at 22-25 weeks GA, and may represent a more appropriate model for the development of an AP system $27,33,34$

Upon initiation of AP support, we observed supraphysiologic UV flow in all pumped AP experiments. Umbilical vein blood flow was higher in fetuses supported with a pumped circuit than in fetuses supported using a pumpless system. Our experiments revealed a positive correlation between fetal HR and UV flow on the pumped AP, with persistent tachycardia seen with both systems. Contrary to our speculations regarding supraphysiologic resistance in the pumpless circuit ${ }^{27}$, we propose that the negative pressure generated by the centrifugal pump may have diminished right ventricular afterload and minimized resistance to flow across the UA cannulas, resulting in higher than normal flow rates. The association we observed between fetal HR and UV flow in animals supported on the pumped circuit may be attributable to the Frank-Starling mechanism and Anrep effect ${ }^{35}$, whereby increased ventricular preload enhances myocardial stretch and tension, resulting in increased contractility. Although this relationship holds true for slight increases in end-diastolic filling pressures, the fetal heart has little preload reserve ${ }^{36-39}$. Thus, further augmentation of cardiac output and contractility are also driven by the Bowditch effect ${ }^{40}$ through increases in fetal HR $^{41}$. In contrast to the physiology seen in a pumpless circuit, we observed a shift of placental-to-systemic blood flow in favor of the AP, effectively volume loading the fetal heart. Therefore, we speculate that these supraphysiologic circuit flows lead to increased preload, tachycardia, sympathetic nervous system activation, and likely higher cardiac output within the first hours of AP support.

After 24 hours of AP support, we observed marked reductions in UV flow compared to the high flows seen at the start of AP support and in many experiments, UV flows then remained subphysiologic. The drop in UV blood flow and persistent tachycardia could be explained by increased sympathetic nervous activation and continuous peripheral vasoconstriction. Although the introduction of milrinone improved UV flow and reduced BP, its inotropic and systemic vasodilatory effects appeared to be short-lived, indicating the presence of overwhelming perturbation of fetal cardiac loading conditions. However, our findings suggest that phosphodiesterase inhibitors may have a positive impact on the circulation of fetal animals supported on the $\mathrm{AP}^{19,42}$. Despite similar $\mathrm{SO}_{2}$, blood gases showed higher $\mathrm{PO}_{2}$, lower $\mathrm{PCO}_{2}$, as well as a more alkalotic $\mathrm{pH}$ in fetuses on the pumped AP compared to in utero controls. We were able to manage $\mathrm{O}_{2}$ uptake and $\mathrm{CO}_{2}$ elimination better on the pumped circuit than pumpless. However, the gas exchange capacity of the oxygenator used in both groups easily outperforms the native placenta, resulting in supraphysiologic UV $\mathrm{PO}_{2}$. High oxygen tension is a known contributor to umbilical cord and ductal constriction and may have negatively affected our experiments ${ }^{43,44}$. Gradual umbilical vessel spasm or constriction at the umbilicus, where the cord passes through the abdominal wall, could explain the gradual decline in UV flow we observed. Mean blood pressure in the UV is 6-8 mmHg in near-term sheep and human fetuses ${ }^{29,30}$. In our pumped AP studies, we observed pre- and post-oxygenator pressures of $25 \mathrm{mmHg}$ and $18 \mathrm{mmHg}$, respectively (Supplementary Figure 1.). Although we did not measure blood pressure in the UV, it is likely that the pumped circuit exposed the umbilical vein to elevated blood pressure, even if there was some degree of pressure difference across the venous cannula. Berman et al. ${ }^{45}$ advanced balloon catheters into the common UV of fetal sheep and demonstrated marked reductions in placental blood flow with increasing UV pressures. This was attributed to a decreased pressure gradient across the umbilical circulation, resulting in diminished umbilical flows. We hypothesize that a similar phenomenon may be occurring in the early phase of our AP experiments, with UV constriction induced by the high pressures and shear stress resulting from the elevated post-pump pressures.

We observed a steady widening of the veno-arterial saturation gradient across the umbilical circulation during our AP runs that was negatively correlated with UV flow, indicating higher oxygen extraction at the tissues in the setting of a net reduction in UV flow and oxygen delivery. One factor that might additionally contribute to the progressive reduction in oxygen delivery was the low hemoglobin concentration we observed in animals supported on the pumped system. The large priming volume of the circuit and oxygenator contributes to fetal anemia because the circuit is primed with maternal blood. During pregnancy, maternal blood volume increases by $\sim 40 \%$ and there is a progressive decline in hemoglobin concentrations ${ }^{46-48}$. Conversely, fetal hemoglobin increases with $\mathrm{GA}$ to sustain fetal tissue oxygenation in the setting of declining $\mathrm{PO}_{2}{ }^{29,49,50}$. Thus, the mixing of maternal and fetal blood in the AP circuit contributes to hemodilution of fetal blood, while ongoing blood sampling further depletes fetal red blood cells. To circumvent this problem in future experiments, it may be possible to hemoconcentrate the circuit prime. 
Furthermore, pump-induced hemolysis may have also contributed to fetal anemia in these experiments. Other groups have recommended the administration of daily doses of erythropoietin to minimize the need for successive maternal donor blood transfusions ${ }^{17}$. Miniaturization of the oxygenator and pump head could also help to reduce fetal anemia by reducing circuit volume.

\section{Study limitations.}

There are several limitations of the present study. Firstly, we have not yet characterized the etiology of the variation in UV flows on the pumped AP circuit. We speculate that a combination of UV constriction and supraphysiologic UV pressures, as well as supraphysiologic oxygen tension may be contributing to progressively diminished UV flows. However, this hypothesis should be tested with direct measurements of UV pressure. Similarly, future experiments would be strengthened by the simultaneous measurement of umbilical artery pressure. We speculate that adrenergic drive may be contributing to tachycardia and peripheral vasoconstriction on the AP that would be enhanced by the measurement of circulating catecholamines. Secondly, although the addition of a centrifugal pump to the AP improved fetal pig hemodynamics, we periodically encountered air-entrainment into the circuit, as well as cavitation of fetal blood at excessively high negative pressures, resulting in diminished circuit flows. Although we did not detect pump-induced hemolysis, this could be a limiting factor during long-term AP experiments and could explain the fetal anemia on the circuit. Finally, systemic inflammation may have also contributed to the hemodynamic instability and cardiovascular decompensation we observed in pumped AP fetal pigs as centrifugal pumps have been reported to induce low-level systemic inflammation ${ }^{51}$. The analysis of inflammatory cytokines may be helpful to delineate the role of inflammation in fetal pig circulatory physiology on the AP.

\section{Future directions.}

Recent developments in AP technology have enabled sustained extrauterine fetal life on a pumpless arteriovenous ECMO circuit. Of note, these experiments were conducted using customized hollow-fiber membrane oxygenators that are unavailable for commercial use. Our initial approach in developing a pumpless AP system involved cannulas with the largest luminal diameter to wall thickness ratio possible to minimize circuit resistance. Our failure to demonstrate hemodynamic stability using a pumpless circuit encouraged our team to explore the use of mechanical support in the extremely preterm fetal pig. While the addition of a pump to the circuit improved survival, we observed supraphysiologic circuit flows, evidence of adrenergic drive, and high cardiac output at the start of support, followed by diminished UV flows with persistent tachycardia and hypertension in keeping with diminished cardiac output. This pattern may indicate a progressive increase in circuit resistance resulting from constriction in the cord vessels. An alternative approach to normalizing UV pressures and minimizing the pressure difference across the UV cannula would be to introduce a pressure drop in the circuit through restriction of the circuit or cannula lumen. According to Hagan-Poiseuille's law, reducing the calibre of the venous side of the circuit would increase circuit resistance and limit the supraphysiologic circuit blood flow seen at the onset of our AP experiments.

Miniaturization of the oxygenator and pump head may also help to achieve hemodynamic stability in our animal model. In sheep, placental blood volume at $\sim 116$ days GA (term $=145$ days) is $\sim 60-80 \mathrm{ml} / \mathrm{kg}$, which represents $\sim 30-40 \mathrm{ml}$ for a $0.5 \mathrm{~kg}$ fetus ${ }^{52,53}$. The AP circuit used in the present study has a priming volume that is nearly double the expected placental blood volume for a fetal sheep delivered at 116 days gestation, which likely accounts for the increased afterload we seemed to induce in our pumpless AP experiments, as well as contributing to fetal anemia. Reductions in the oxygenator membrane surface area could also reduce the need for excessive heparin administration. Thus, modifications to the circuit through miniaturization of the oxygenator and centrifugal pump may be helpful for establishing an AP system capable of supporting the long-term physiological requirements of extremely preterm fetal pigs.

\section{Conclusion}

Over the past decade, important advances have been achieved in the field of artificial womb/AP technology. In experiments using a miniature pig model of the AP in which commercial neonatal ECMO oxygenators were connected the fetal circulation via the umbilical cord we demonstrated a marked improvement in survival, UV flow, and oxygen delivery in circuits that incorporated a small centrifugal pump compared with animals maintained on a pumpless circuit. However, despite the addition of the pump, we observed a progressive diminution of UV flow with persistent tachycardia and hypertension, which we attribute to preload imbalance, increased sympathetic tone, and UV hypertension. Thus, despite observing a clear short-term benefit with the addition of a centrifugal pump in supporting the fetal pig hemodynamics, we conclude that further modifications to the AP circuit are needed. A reduction to the size of the UV cannula could represent an alternative approach for mediating physiologic circuit flows and venous pressures. Given the limitations of neonatal intensive care therapies in preventing iatrogenic organ injury and neonatal death, we remain hopeful that the AP could provide reductions in the mortality and morbidity associated with preterm birth by maintaining a fetal circulation while allowing for normal growth and development of fetal organ systems.

\section{Declarations}

\section{Competing interests}

The authors have no competing interests to disclose. 
Author Contributions

Conception or design of the work: MS, JLM, CH.

Data acquisition: AJCP, SKSC, TA, LS, AAF, MJM, AS, ME, LCL, CF, DM, JB, BSS, JL, OJM, FL, JRTD, MS, JLM, CH.

Data analysis or interpretation: AJCP, SKSC, TA, AAF, MJM, MQ, JRTD, MS, JLM, CH.

Drafting the work or revising it critically for important intellectual content: AJCP, SKSC, TA, LS, AAF, MJM, JB, JRTD, MS, JLM, CH.

All authors approved the final version of the manuscript, agree to be accountable for all aspects of the work in ensuring that questions related to the accuracy or integrity of any part of the work are appropriately investigated and resolved, and all persons designated as authors qualify for authorship, and all those who qualify for authorship are listed.

\section{Funding}

The artificial placenta experiments were generously supported by funding from the Canadian Institutes of Health Research and Breakfast of Champions and BMO Financial Group Chair in Cardiology to MS.

Landrace pig studies and JLM were funded by an ARC Future Fellowship (Level 3; FT170100431). BSS was funded by SickKids Research Institute's graduate scholarship program, Restracomp.

\section{Data availability}

The data that support the findings of this study are available from the corresponding author upon reasonable request.

\section{Acknowledgements}

The authors would like to acknowledge Luke Itani for his illustrations of Figures 1 and Supplementary Figure 1. The authors would also like to acknowledge the staff members in the Lab Animal Services at The Hospital for Sick Children, Peter Gilgan Center for Research and Learning, Early Origins of Adult Health Research Group and PIRL, SAHMRI.

\section{References}

1. Patel, R. M. et al. Causes and Timing of Death in Extremely Premature Infants from 2000 through 2011. N. Engl. J. Med. 372, 331-340 (2015).

2. Matthews, T. J., MacDorman, M. F. \& Thoma, M. E. Infant Mortality Statistics From the 2013 Period Linked Birth/Infant Death Data Set. Natl. Vital Stat. Rep. Cent. Dis. Control Prev. Natl. Cent. Health Stat. Natl. Vital Stat. Syst. 64, 1-30 (2015).

3. Stoll, B. J. et al. Neonatal Outcomes of Extremely Preterm Infants From the NICHD Neonatal Research Network. PEDIATRICS 126, $443-456$ (2010).

4. Blencowe, H. et al. Born Too Soon: The global epidemiology of 15 million preterm births. Reprod. Health 10, S2 (2013).

5. Lee, S. K. et al. Outcomes and care practices for preterm infants born at less than 33 weeks' gestation: a quality-improvement study. CMAJ Can. Med. Assoc. J. J. Assoc. Medicale Can. 192, E81-E91 (2020).

6. Thébaud, B. et al. Bronchopulmonary dysplasia. Nat. Rev. Dis. Primer 5, 78 (2019).

7. Wood, N. S., Marlow, N., Costeloe, K., Gibson, A. T. \& Wilkinson, A. R. Neurologic and developmental disability after extremely preterm birth. EPICure Study Group. N. Engl. J. Med. 343, 378-384 (2000).

8. Westin, B., Nyberg, R. \& Enhörning, G. A Technique for Perfusion of the Previable Human Fetus. Acta Paediatr. 47, 339-349 (1958).

9. Callaghan, J. C., Maynes, E. A. \& Hug, H. R. STUDIES ON LAMBS OF THE DEVELOPMENT OF AN ARTIFICIAL PLACENTA. REVIEW OF NINE LONG-TERM SURVIVORS OF EXTRACORPOREAL CIRCULATION MAINTAINED IN A FLUID MEDIUM. Can. J. Surg. J. Can. Chir. 8, $208-213$ (1965).

10. De Bie, F. R., Davey, M. G., Larson, A. C., Deprest, J. \& Flake, A. W. Artificial placenta and womb technology: Past, current, and future challenges towards clinical translation. Prenat. Diagn. 41, 145-158 (2021).

11. Zapol, W. M., Kolobow, T., Pierce, J. E., Vurek, G. G. \& Bowman, R. L. Artificial Placenta: Two Days of Total Extrauterine Support of the Isolated Premature Lamb Fetus. Science 166, 617-618 (1969).

12. Reoma, J. L. et al. Development of an artificial placenta I: pumpless arterio-venous extracorporeal life support in a neonatal sheep model. J. Pediatr. Surg. 44, 53-59 (2009).

13. Miura, Y. et al. Novel modification of an artificial placenta: pumpless arteriovenous extracorporeal life support in a premature lamb model. Pediatr. Res. 72, 490-494 (2012). 
14. Miura, Y. et al. A Parallelized Pumpless Artificial Placenta System Significantly Prolonged Survival Time in a Preterm Lamb Model: Parallelization of Artificial Placenta System. Artif. Organs 40, E61-E68 (2016).

15. Gray, B. W. et al. Development of an artificial placenta V: 70h veno-venous extracorporeal life support after ventilatory failure in premature lambs. J. Pediatr. Surg. 48, 145-153 (2013).

16. Schoberer, M. et al. Miniaturization: The Clue to Clinical Application of the Artificial Placenta: Miniaturization of the Artificial Placenta. Artif. Organs 38, 208-214 (2014).

17. Partridge, E. A. et al. An extra-uterine system to physiologically support the extreme premature lamb. Nat. Commun. 8, 15112 (2017).

18. Usuda, H. et al. Successful maintenance of key physiological parameters in preterm lambs treated with ex vivo uterine environment therapy for a period of 1 week. Am. J. Obstet. Gynecol. 217, 457.e1-457.e13 (2017).

19. Usuda, H. et al. Successful use of an artificial placenta to support extremely preterm ovine fetuses at the border of viability. Am. J. Obstet. Gynecol. 221, 69.e1-69.e17 (2019).

20. Church, J. T. et al. Perfluorocarbons Prevent Lung Injury and Promote Development during Artificial Placenta Support in Extremely Premature Lambs. Neonatology 113, 313-321 (2018).

21. Church, J. T. et al. Effects of an artificial placenta on brain development and injury in premature lambs. J. Pediatr. Surg. 53, 1234-1239 (2018).

22. El-Sabbagh, A. M. et al. Cerebral Oxygenation of Premature Lambs Supported by an Artificial Placenta: ASAIO J. 64, 552-556 (2018).

23. Hornick, M. A. et al. Umbilical cannulation optimizes circuit flows in premature lambs supported by the EXTra-uterine Environment for Neonatal Development (EXTEND): Umbilical cannulation optimizes circuit flows on EXTEND. J. Physiol. 596, 1575-1585 (2018).

24. Coughlin, M. A. et al. An Artificial Placenta Protects Against Lung Injury and Promotes Continued Lung Development in Extremely Premature Lambs: ASAIO J. 65, 690-697 (2019).

25. Schittny, J. C. Development of the lung. Cell Tissue Res. 367, 427-444 (2017).

26. Morrison, J. L. et al. Improving pregnancy outcomes in humans through studies in sheep. Am. J. Physiol.-Regul. Integr. Comp. Physiol. 315, R1123-R1153 (2018).

27. Charest-Pekeski, A. J. et al. Achieving sustained extrauterine life: Challenges of an artificial placenta in fetal pigs as a model of the preterm human fetus. Physiol. Rep. 9, (2021).

28. Darby, J. R. T. et al. Haemodynamics and cerebral oxygenation of neonatal piglets in the immediate ex utero period supported by mechanical ventilation or ex utero oxygenator. J. Physiol. 599, 2751-2761 (2021).

29. Acharya, G., Sonesson, S.-E., Flo, K., Räsänen, J. \& Odibo, A. Hemodynamic aspects of normal human feto-placental (umbilical) circulation. Acta Obstet. Gynecol. Scand. 95, 672-682 (2016).

30. Rudolph, A. M. Congenital diseases of the heart: clinical-physiological considerations. (Wiley-Blackwell, 2009).

31. Kuwabara, Y. et al. Development of Extrauterine Fetal Incubation System Using Extracorporeal Membrane Oxygenator. Artif. Organs 11, 224227 (1987).

32. Hornick, M. A. et al. Technical feasibility of umbilical cannulation in midgestation lambs supported by the EXTra-uterine Environment for Neonatal Development (EXTEND). Artif. Organs 43, 1154-1161 (2019).

33. Chan, K. Y. Y., Miller, S. L., Schmölzer, G. M., Stojanovska, V. \& Polglase, G. R. Respiratory Support of the Preterm Neonate: Lessons About Ventilation-Induced Brain Injury From Large Animal Models. Front. Neurol. 11, 862 (2020).

34. Eiby, Y. A. et al. A Pig Model of the Preterm Neonate: Anthropometric and Physiological Characteristics. PLoS ONE 8, e68763 (2013).

35. von Anrep, G. On the part played by the suprarenals in the normal vascular reactions of the body. J. Physiol. 45, 307-317 (1912).

36. Kirkpatrick, S., Pitlick, P., Naliboff, J. \& Friedman, W. Frank-Starling relationship as an important determinant of fetal cardiac output. Am. J. Physiol.-Leg. Content 231, 495-500 (1976).

37. Gilbert, R. D. Control of fetal cardiac output during changes in blood volume. Am. J. Physiol.-Heart Circ. Physiol. 238, H80-H86 (1980).

38. Thornburg, K. L. \& Morton, M. J. Filling and arterial pressures as determinants of left ventricular stroke volume in fetal lambs. Am. J. Physiol. 251, H961-968 (1986).

39. Rychik, J. Fetal Cardiovascular Physiology. Pediatr. Cardiol. 25, (2004).

40. Piot, C. et al. High Frequency-Induced Upregulation of Human Cardiac Calcium Currents. Circulation 93, 120-128 (1996).

41. Kiserud, T. \& Acharya, G. The fetal circulation. Prenat. Diagn. 24, 1049-1059 (2004).

42. De Bie, F. R. et al. Pharmacokinetics and pharmacodynamics of sildenafil in fetal lambs on extracorporeal support. Biomed. Pharmacother. 143,112161 (2021).

43. Eltherington, L. G., Stoff, J., Hughes, T. \& Melmon, K. L. Constriction of human umbilical arteries. Interaction between oxygen and bradykinin. Circ. Res. 22, 747-752 (1968). 
44. McGrath, J. C., MacLennan, S. J., Mann, A. C., Stuart-Smith, K. \& Whittle, M. J. Contraction of human umbilical artery, but not vein, by oxygen. J. Physiol. 380, 513-519 (1986).

45. Berman, W., Goodlin, R. C., Heymann, M. A. \& Rudolph, A. M. Relationships between pressure and flow in the umbilical and uterine circulations of the sheep. Circ. Res. 38, 262-266 (1976).

46. Rockwell, L. C., Vargas, E. \& Moore, L. G. Human physiological adaptation to pregnancy: inter- and intraspecific perspectives. Am. J. Hum. Biol. Off. J. Hum. Biol. Counc. 15, 330-341 (2003).

47. Means, R. T. Iron Deficiency and Iron Deficiency Anemia: Implications and Impact in Pregnancy, Fetal Development, and Early Childhood Parameters. Nutrients 12, 447 (2020).

48. Gonzales, G. F., Steenland, K. \& Tapia, V. Maternal hemoglobin level and fetal outcome at low and high altitudes. Am. J. Physiol. Regul. Integr. Comp. Physiol. 297, R1477-1485 (2009).

49. Jopling, J., Henry, E., Wiedmeier, S. E. \& Christensen, R. D. Reference ranges for hematocrit and blood hemoglobin concentration during the neonatal period: data from a multihospital health care system. Pediatrics 123, e333-337 (2009).

50. Society for Maternal-Fetal Medicine (SMFM). Electronic address: pubs@smfm.org et al. Society for Maternal-Fetal Medicine (SMFM) Clinical Guideline \#8: the fetus at risk for anemia-diagnosis and management. Am. J. Obstet. Gynecol. 212, 697-710 (2015).

51. Ugaki, S. et al. Efficacy of a Miniature Centrifugal Rotary Pump (TinyPump) for Transfusion-Free Cardiopulmonary Bypass in Neonatal Piglets. ASAIO J. Am. Soc. Artif. Intern. Organs 1992 53, 675-9 (2007).

52. Brace, R. A. Blood volume and its measurement in the chronically catheterized sheep fetus. Am. J. Physiol.-Heart Circ. Physiol. (1983) doi:10.1152/ajpheart.1983.244.4.H487.

53. Yao, A., Moinian, M. \& Lind, J. DISTRIBUTION OF BLOOD BETWEEN INFANT AND PLACENTA AFTER BIRTH. The Lancet 294, $871-873$ (1969).

54. Kilkenny, C., Browne, W. J., Cuthill, I. C., Emerson, M. \& Altman, D. G. Improving bioscience research reporting: the ARRIVE guidelines for reporting animal research. PLoS Biol. 8, e1000412 (2010).

55. Schrauben, E. M. et al. Open or closed: Changes in ductus arteriosus flow patterns at birth using 4D flow MRI in newborn piglets. Physiol. Rep. 9, e14999 (2021).

\section{Figures}

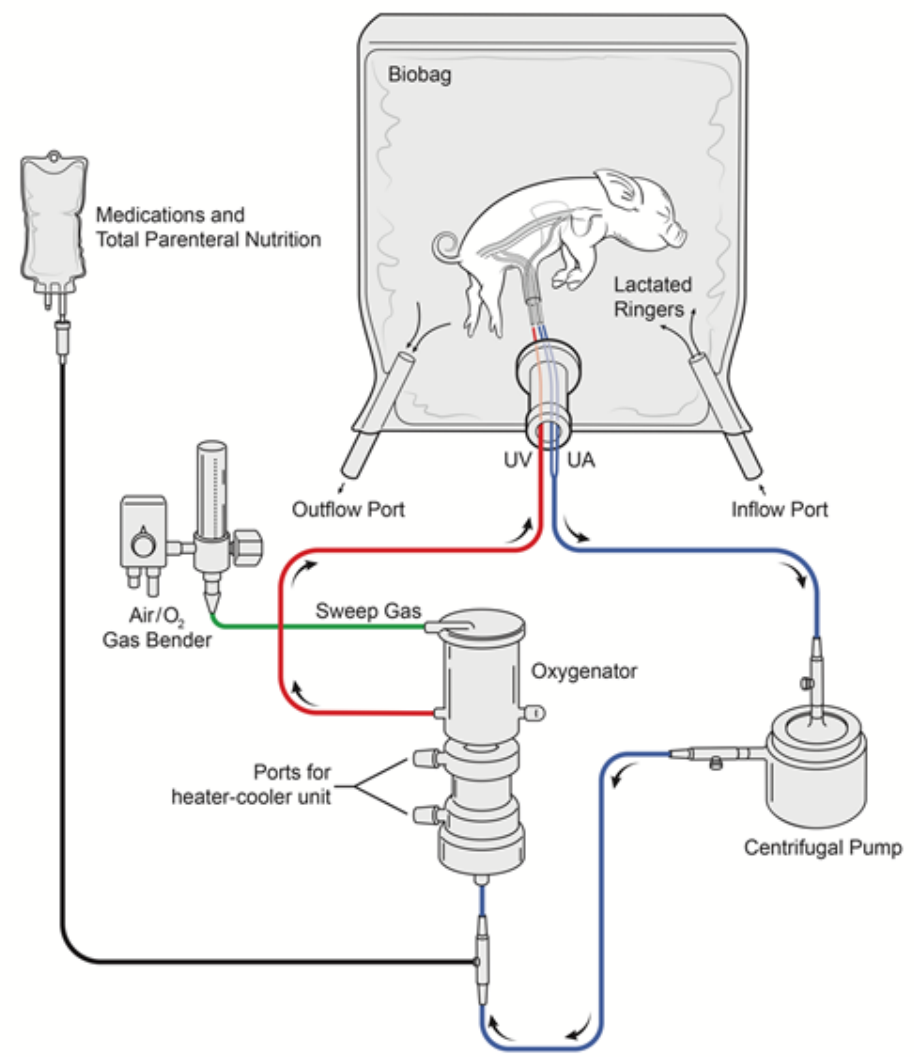

Figure 1 
Layout of the pumped AP circuit. Fetal pigs $(n=13)$ were delivered via caesarean section, cannulated via the UA's and UV, and maintained using a pumped AP circuit. Fetal pigs ( $n=12$ ) were also maintained using the same circuit design but without a centrifugal pump. Blood passed through the UAs and the centrifugal pump before entering the inflow port of the oxygenator. Oxygenated blood then passes through the oxygenator outflow port before returning to the heart via the UV. Sweep gas supplied the oxygenator with medical air and oxygen and the Biobag was filled with warmed LR solution to maintain fetal normothermia.

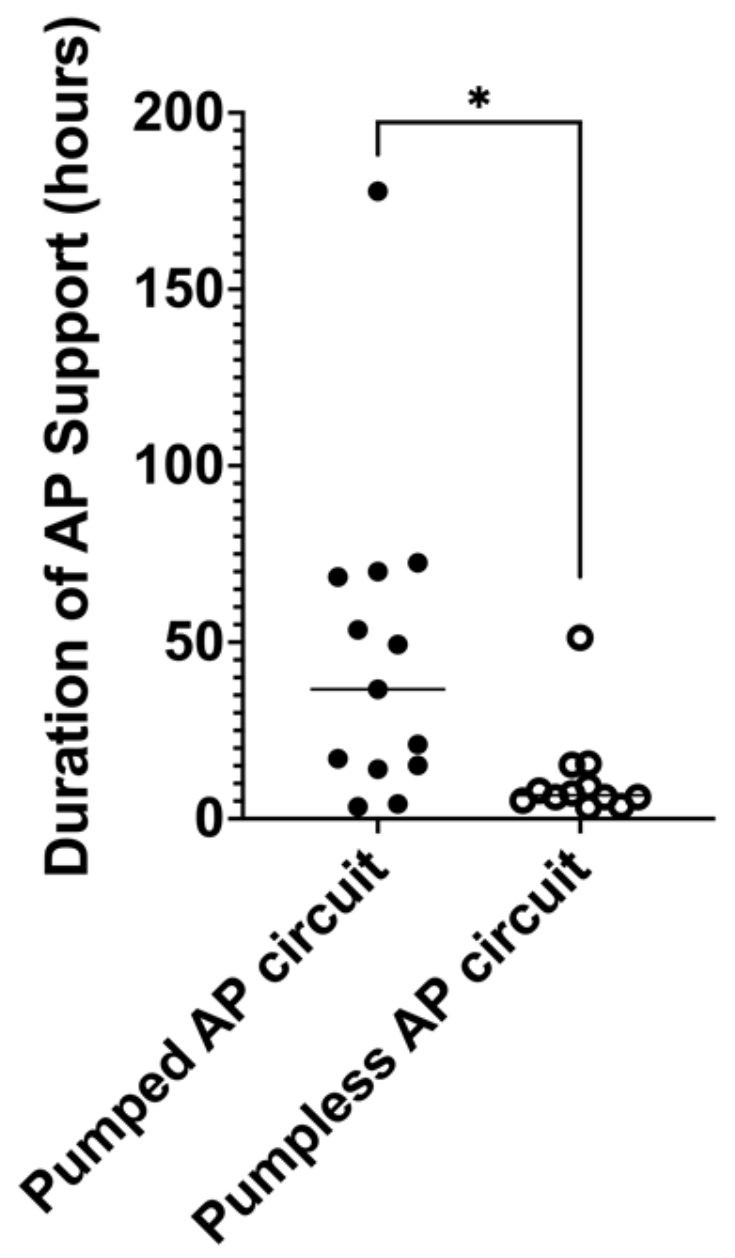

Figure 2

Duration of AP support between fetal pigs supported on a pumped and pumpless ${ }^{27}$ AP circuit. Fetal pigs maintained using a pumped AP circuit are shown as black circles $(n=13)$, and fetuses supported using a pumpless circuit as open circles $(n=12)$. 

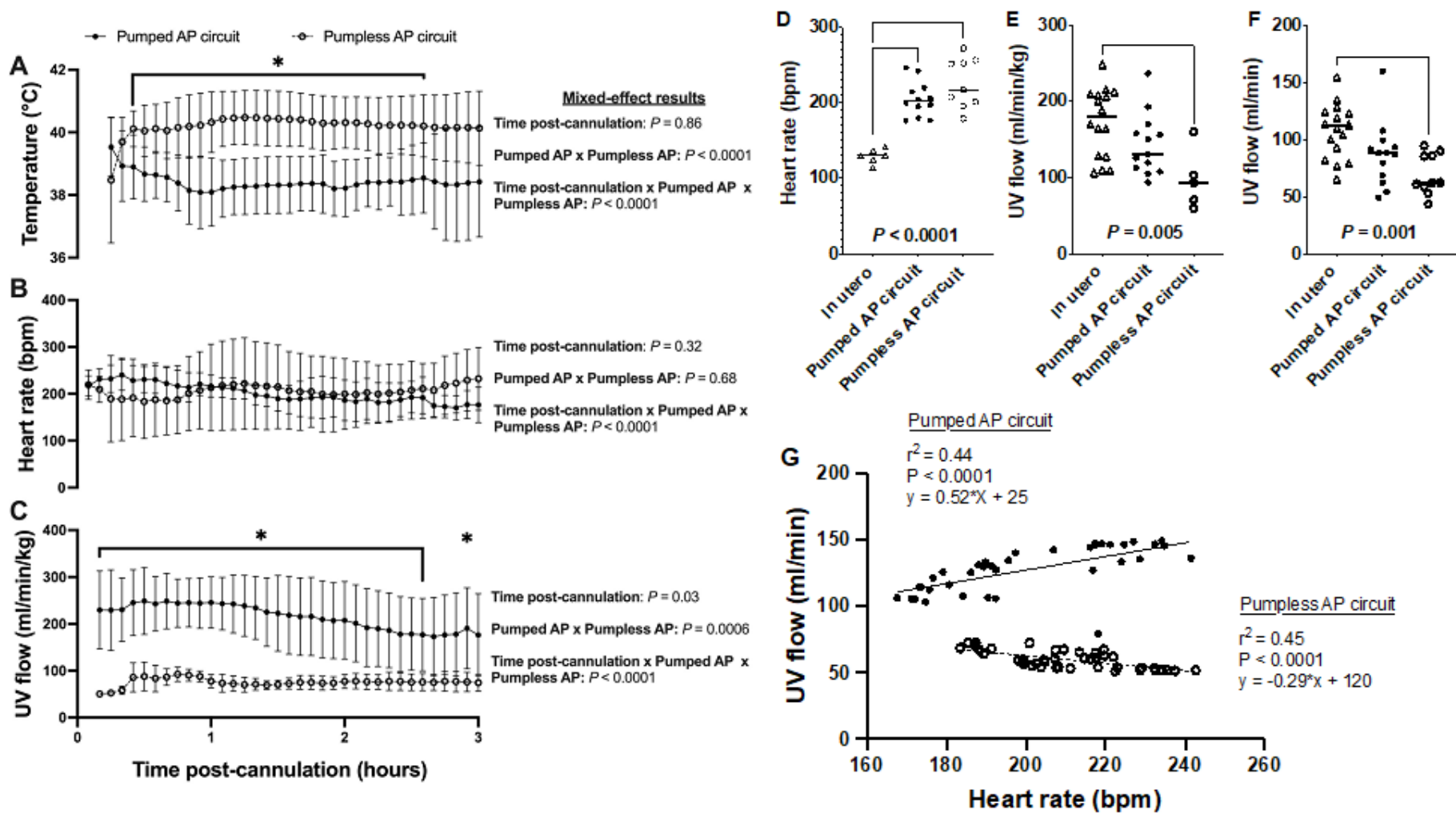

Figure 3

Fetal temperature, HR, and UV flow data for in utero, pumped and pumpless AP fetal pigs ${ }^{27}$. (A) Temperature; (B) Fetal HR; and (C) UV flow vs. time post-cannulation. (D) Mean fetal HR; (E) mean indexed UV flow; and (F) mean absolute UV flow over the entire duration of support. (G) Correlations between HR and absolute UV flow for fetal pigs supported using a pumpless and pumped AP circuit. Fetal pigs maintained on a pumpless AP are represented as open circles and dashed lines ( $n=10$ for temperature; $n=9$ for HR; $n=5$ for UV flow), and fetal pigs maintained on a pumped AP circuit ( $n=13$ for temperature and UV flow; $n=12$ for HR) as black circles and solid line. In utero fetal pigs are represented as open triangles ( $n=6$ for HR; $n=16$ for indexed and absolute UV), pumpless AP fetal pigs as open circles ( $n=10$ for absolute UV flow) and pumped AP fetal pigs as black circles $(n=13$ for unindexed UV flow 
\ In utero - Pumped AP circuit o Pumpless AP circuit

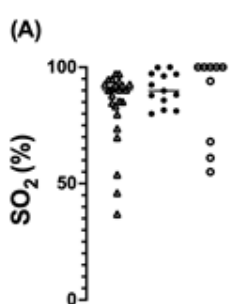

(E)
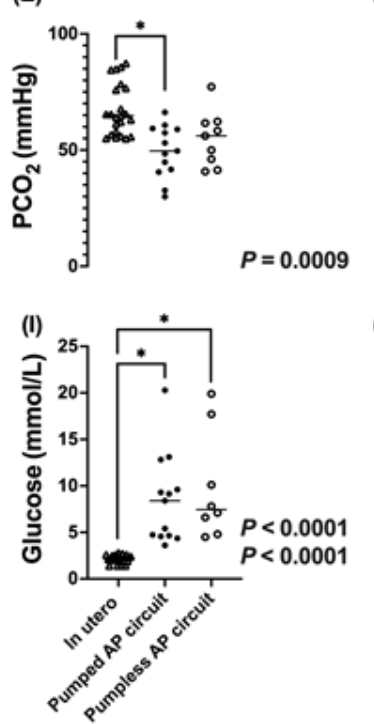

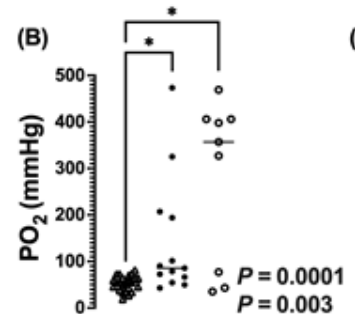

(C)

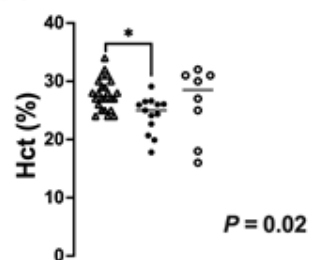

(G)

(F)

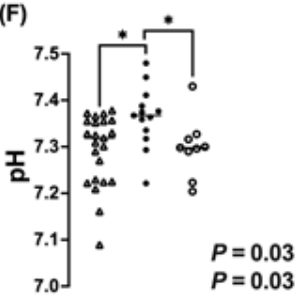

(D)

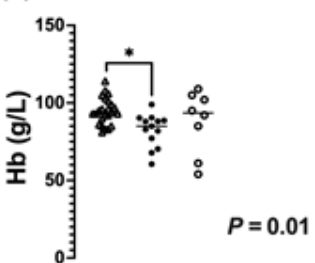

(H)

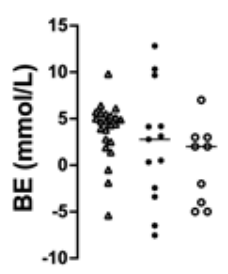

(J)

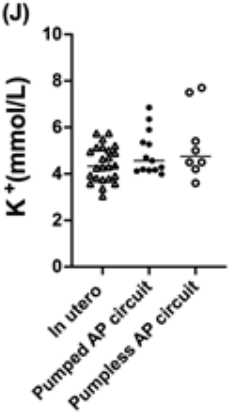

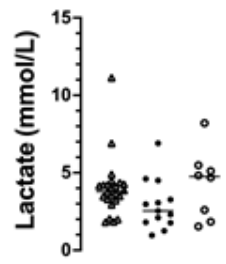

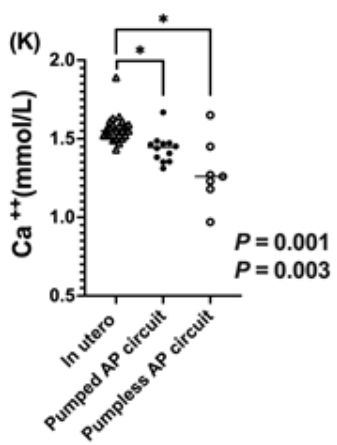

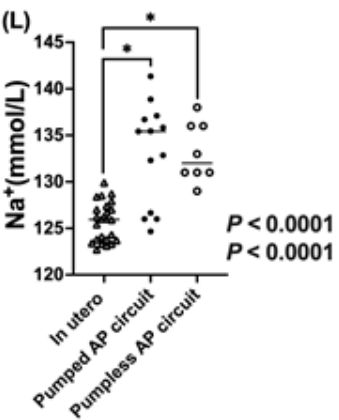

Figure 4

Blood gases, electrolytes, lactate, and glucose concentrations in fetal pigs studied in utero versus fetal pigs supported using a pumped and pumpless AP circuit ${ }^{27}$. In utero fetal pig data represented by open triangles $(n=24)$, fetal pigs supported on a pumped AP circuit as black circles $(n$ $=13)$, and fetuses supported on a pumpless AP circuit as open circles $(n=9)$. If two $P$-values are presented, the first $P$-values represents the pumped AP circuit, and the second $P$-value represents the pumpless AP circuit. 

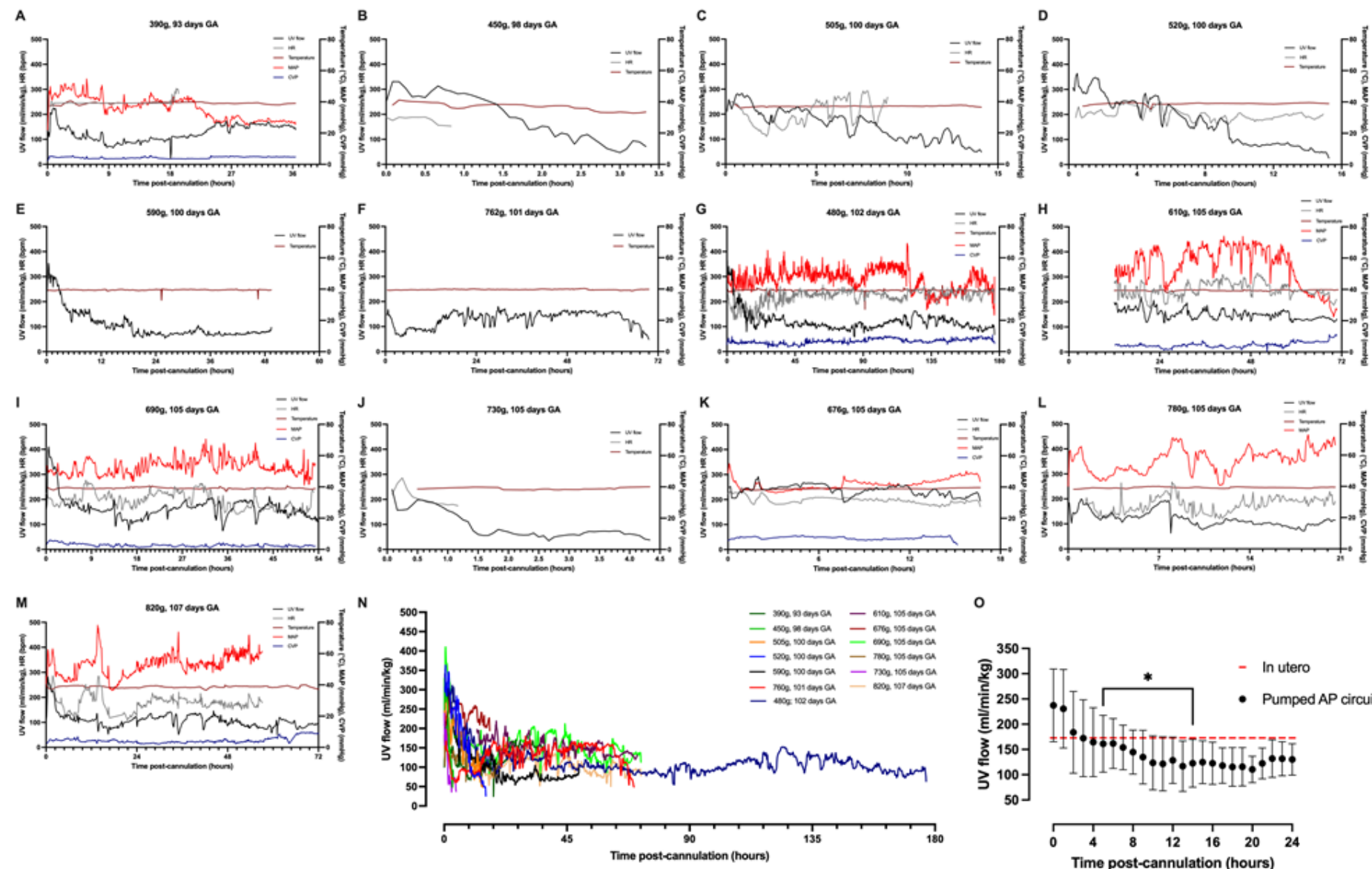

o

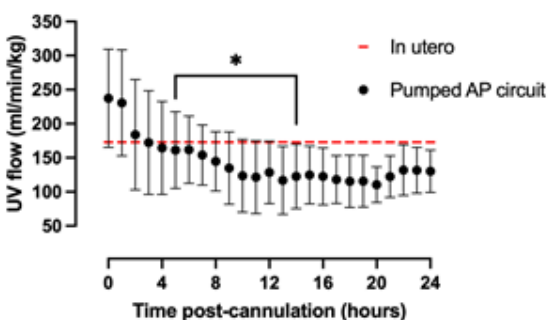

Figure 5

Hemodynamic data in pumped AP fetal pigs. Data from $n=13$ fetal pigs. (A-M) Changes in UV flow, HR, temperature, MAP, and CVP over time for each individual animal supported using a pumped AP circuit. ( $N$ ) Individual UV flow patterns for fetal pigs. (0) Aggregate UV flow data for pumped AP fetal pigs. Pumped AP fetal pigs are represented as black circles, and in utero UV flow as the dashed grey line. Data are presented in 5-minute $(\mathrm{A}-\mathrm{N})$ and 1 hour epochs (0). * Significantly different from the first recorded data point.

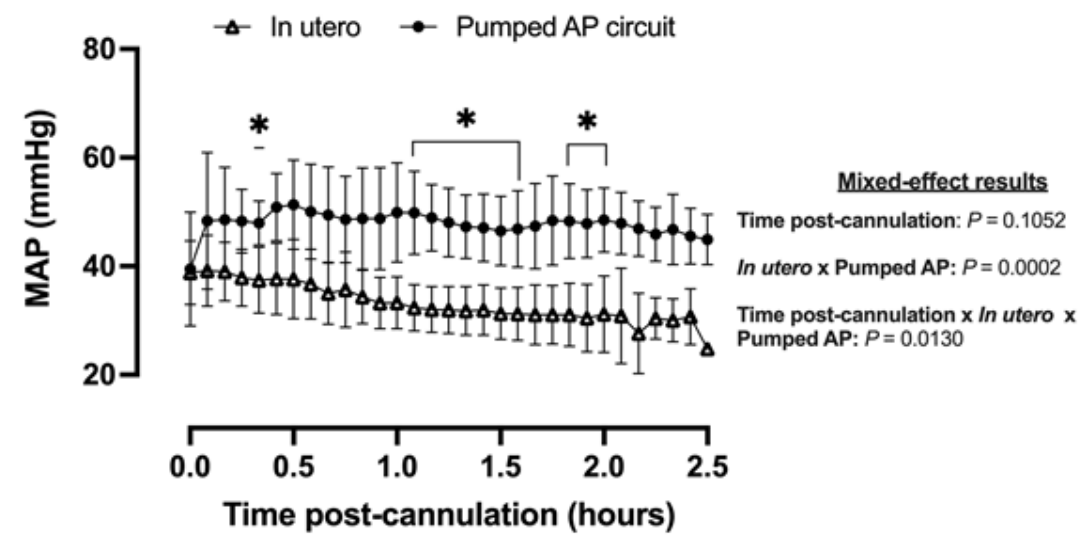

Figure 6

MAP in fetal pigs studied in utero and maintained using a pumped AP circuit. Fetal pigs studied in utero are represented in open triangles ( $\mathrm{n}=21$ ) and fetal pigs maintained using a pumped AP as black circles $(n=6)$. 

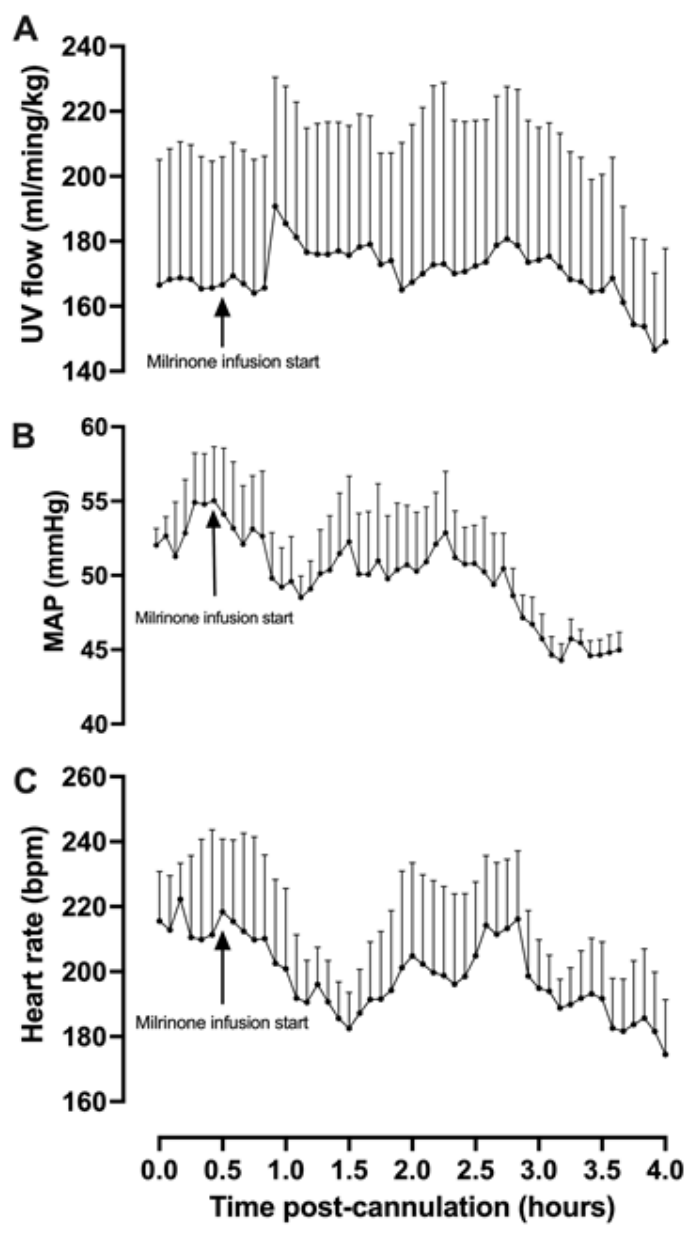

Figure 7

Effects of milrinone lactate infusion on fetal pig hemodynamics while maintained using a pumped AP circuit. Maintenance infusion of milrinone lactate was used in successful AP experiments $(n=5)$ for hemodynamic support. 'Milrinone infusion start' indicates the time point at which milrinone lactate IV infusion began. Data are presented as 5-minute epochs and expressed as mean \pm SEM.

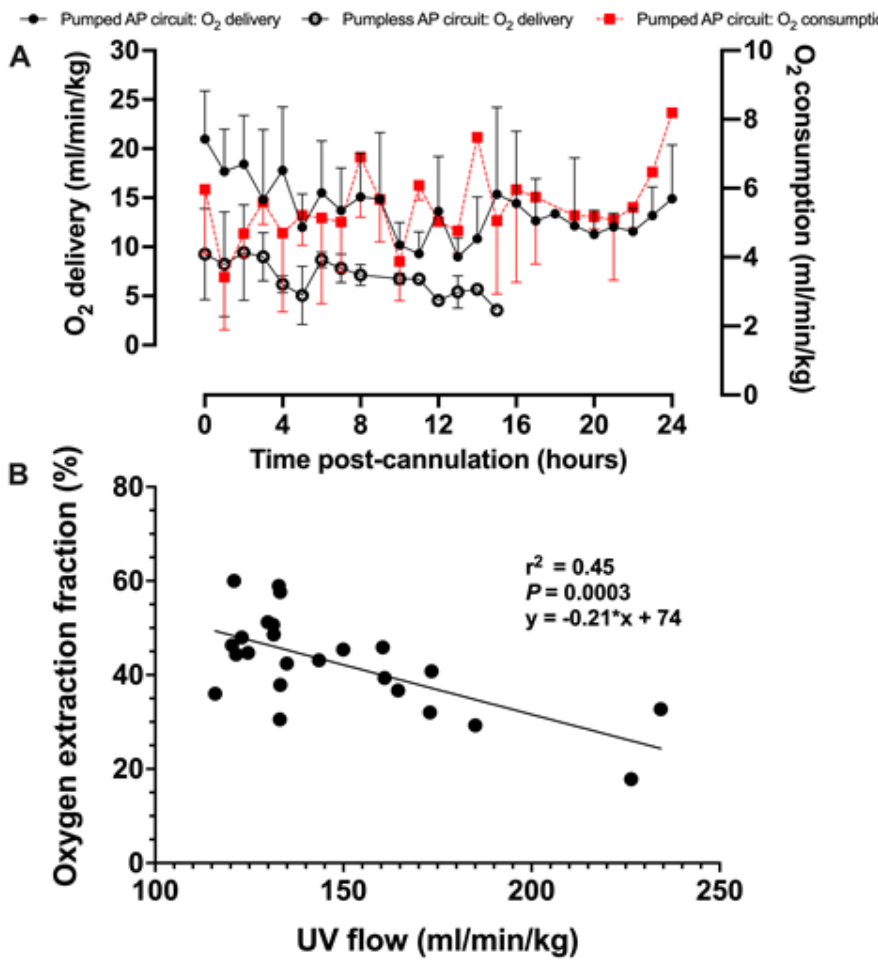


Figure 8

Fetal oxygen delivery, oxygen consumption, and oxygen extraction fraction on a pumped AP circuit. (A) Fetal oxygen delivery on the pumped AP circuit is represented as black circles $(n=13)$ and open circles for the pumpless AP circuit $(n=5)^{27}$, whereas oxygen consumption is shown as red squares with a dashed line $(n=11)$. (B) Correlation between UV flow and oxygen extraction fraction $(n=11)$. Data are presented as 1 hour averages over the first 24 hours of AP support.

\section{Supplementary Files}

This is a list of supplementary files associated with this preprint. Click to download.

- SupplementaryFigure1PumpedAPPaper.pdf 